\title{
Phase Transitions of Binary Lipid Mixtures: A Combined Study by Adiabatic Scanning Calorimetry and Quartz Crystal Microbalance with Dissipation Monitoring
}

\author{
P. Losada-Pérez, ${ }^{1,2}$ N. Mertens, ${ }^{3}$ B. de Medio-Vasconcelos, ${ }^{4}$ \\ E. Slenders, ${ }^{3}$ J. Leys, ${ }^{3}$ M. Peeters, ${ }^{1}$ B. van Grinsven, ${ }^{5}$ J. Gruber, ${ }^{4}$ C. Glorieux, ${ }^{3}$ \\ H. Pfeiffer, ${ }^{6}$ P. Wagner, ${ }^{1,2}$ and J. Thoen ${ }^{3}$ \\ ${ }^{1}$ Institute for Materials Research IMO, Hasselt University, Wetenschapspark 1, 3590 Diepenbeek, Belgium \\ ${ }^{2}$ Division IMOMEC, IMEC vzw, Wetenschapspark 1, 3590 Diepenbeek, Belgium \\ ${ }^{3}$ Laboratorium voor Akoestiek en Thermische Fysica, Departement Natuurkunde en Sterrenkunde, KU Leuven, \\ Celestijnenlaan 200D Bus 2416, 3001 Leuven, Belgium \\ ${ }^{4}$ Instituto de Química, Universidade de São Paulo, 748 Av. Prof. Lineu Prestes, 05508-000 São Paulo, SP, Brazil \\ ${ }^{5}$ Maastricht Science Programme, Maastricht University, 6200 MD Maastricht, Netherlands \\ ${ }^{6}$ Structurele Materialen, Departement Materiaalkunde, KU Leuven, Kasteelpark Arenberg 44 Bus 2450, 3001 Leuven, Belgium
}

Correspondence should be addressed to P. Losada-Pérez; patricia.losadaperez@uhasselt.be

Received 19 November 2014; Accepted 17 December 2014

Academic Editor: Charles Rosenblatt

Copyright (C) 2015 P. Losada-Pérez et al. This is an open access article distributed under the Creative Commons Attribution License, which permits unrestricted use, distribution, and reproduction in any medium, provided the original work is properly cited.

\begin{abstract}
The phase transitions of binary lipid mixtures are studied by a combination of Peltier-element-based adiabatic scanning calorimetry (pASC) and quartz crystal microbalance with dissipation monitoring (QCM-D). pASC, a novel type of calorimeter, provides valuable and unambiguous information on the heat capacity and the enthalpy, whereas QCM-D is proposed as a genuine way of determining phase diagrams by analysing the temperature dependence of the viscosity. Two binary mixtures of phospholipids with the same polar head and differing in the alkyl chain length, DMPC + DPPC and DMPC + DSPC, are discussed. Both techniques give consistent phase diagrams, which compare well with literature results, showing their capability to map the phase behaviour of pure lipids as well as lipid mixtures. This work can be considered as a departure point for further investigations on more complex lipid mixtures displaying relevant phases such as the liquid-ordered phase and solid-lipid interfaces with biologically functional importance.
\end{abstract}

\section{Introduction}

During the last decades, the approach to overcome the complexity of biological membranes is to use simplified biomimetic models mainly consisting of binary or ternary mixtures of lipids $[1,2]$. One of the most debated issues in the biophysics of lipids is lipid miscibility and its relevance to the function and organization of biological membranes. In 1997, Simons and Ikonen renewed the interest in lipid phase studies, suggesting that lateral phase separation in biological membranes could be functionally important [3].
Lipid rafts or ordered lipid domains may play a role in the localization, transport, and function of different proteins. As a matter of fact, the number of studies on the influence of domain partitioning on interactions with peptides and nucleic acids has increased significantly in recent years [47]. In spite of the relevance of lipid-protein interactions, the phase behaviour of the lipid components of the cell membrane is believed to be of major importance to uncover some underlying principles behind the membrane function. From a fundamental viewpoint, the interest in lipid phase behaviour stems from the nature and the thermodynamics 
of phase transitions, the mechanisms behind lipid miscibility and lateral phase separation, and the existence of critical phenomena [8]. This requires the knowledge of static and kinetic thermodynamic properties of pure lipids and lipid mixtures. Characterization and mapping of phase diagrams have been carried out for lipid dispersions using several techniques such as differential scanning calorimetry (DSC) [9], nuclear magnetic resonance (NMR) [10], X-ray scattering [11], fluorescence microscopy [12], laser ultrasonics [13], and ultrasonic velocimetry [14]. Nevertheless, the transition temperatures are usually determined with a large uncertainty due to the typically broad transitions and the limited sensitivity of the experimental setups. In turn, only recently surfacesensitive techniques have explored the phase behaviour of lipid layers in contact with solid surfaces, such as supported lipid bilayers (SLBs) and supported layers of vesicles (SLVs). For SLBs atomic force microscopy $[15,16]$, differential scanning calorimetry $[17,18]$, vibrational spectroscopy [18], and ellipsometry [19] have been employed. For SLVs, experiments are so far restricted to pure lipids using dissipation-mode quartz crystal microbalance and heat-transfer studies [20, 21]. Information about the phase behaviour of supported lipid layers is relevant for lipid-based nanobiotechnology applications such as inmunosensors and lipid nanodevices $[22,23]$.

In this work, we study phase transitions of lipid mixtures by means of Peltier-element-based adiabatic scanning calorimetry (pASC) and quartz crystal microbalance with dissipation monitoring (QCM-D). We test the capability of pASC, a novel type of calorimeter, for measuring biologically relevant samples, for which unambiguous information on thermal properties, namely, the specific heat capacity $c_{p}(T)$ and the specific enthalpy $h(T)$, is obtained. Adiabatic scanning calorimetry is a high-resolution calorimetric technique that enables studies of phase transitions and critical phenomena of condensed and soft matter systems. The exceptional temperature stability in the $\mu \mathrm{K}$ range and the slow scanning rates keep the sample close to thermal and thermodynamic equilibrium, allowing the precise determination of phase diagrams [24]. Using QCM-D, we propose a genuine and simple way of determining phase diagrams by analysing the temperature dependence of the viscosity $\eta(T)$.

Although the variety of lipid mixtures is very broad [25], we focused on phospholipids, the most abundant structural elements present in cell membranes. As a proof of concept of the approach, we have chosen two types of phospholipid mixtures, whose phase behaviour has been studied both experimentally [14, 26-30] and by computer simulations [30-33]. The systems are binary mixtures of saturated phospholipids with the same polar head and differing alkyl chain length, namely, 1,2-dimyristoyl-sn-glycero3-phosphocholine (DMPC, chain length $m=14$, melting temperature $T_{m} \sim 24^{\circ} \mathrm{C}$ ), mixed with 1,2-dipalmitoyl-snglycero-3-phosphocholine (DPPC, $m=16, T_{m} \sim 41^{\circ} \mathrm{C}$ ) and mixed with 1,2-distearoyl-sn-glycero-3-phosphocholine (DSPC, $m=18, T_{m} \sim 55^{\circ} \mathrm{C}$ ). Both mixtures are isomorphous systems (the two lipid components are miscible in both fluid and gel phases), but DMPC + DSPC departs more from ideal mixing behaviour due to the larger difference in alkyl chain length between the two types of phospholipids [27, 33].

\section{Experimental}

2.1. Materials. DMPC, DPPC, and DSPC were purchased from Avanti Polar Lipids (Alabaster, AL). HEPES buffer ( $\mathrm{pH}$ 7.4) consisting of $10 \mathrm{mM}$ HEPES from Fisher Scientific (assay 99\%) and $150 \mathrm{mM} \mathrm{NaCl}$ from Sigma-Aldrich (assay $\geq 99.5 \%$ ) was used for hydration of the dried lipids. Spectroscopic grade chloroform was obtained from Analar (Normapur, assay $99.3 \%$ stabilized with about $0.6 \%$ ethanol).

The lipid or lipid mixture was first dissolved in chloroform; the solvent was then evaporated under a mild flow of nitrogen in a round bottomed flask. The resulting lipid film was kept under vacuum overnight to remove any residual solvent. Afterwards, the lipid was hydrated with HEPES buffer. Hydration to $10 \mathrm{mg} \mathrm{mL}^{-1}$ was carried out under continuous stirring in a temperature-controlled water bath at sufficiently high temperature (around $15 \mathrm{~K}$ above the main phase transition temperature $T_{m}$ ). These $10 \mathrm{mg} \mathrm{mL}^{-1}$ samples consisting of mainly multilamellar vesicles were directly used for pASC measurements, while the remaining lipid suspension was diluted to $0.5 \mathrm{mg} \mathrm{mL}^{-1}$ for the QCM-D experiments. Small unilamellar vesicles (SUVs) were formed by extrusion through a filter (Avanti Polar Lipids) with a pore size of $100 \mathrm{~nm}$ for 21 times. Vesicle effective sizes and polydispersities were determined by dynamic light scattering (Zeta Pals, Brookhaven Instruments Corporation). The obtained average diameter was $\sim(130 \pm 30) \mathrm{nm}$. The vesicle dispersions were stored at $4^{\circ} \mathrm{C}$ and used within two days.

For DMPC + DSPC, mixtures with a mole fraction step of 0.1 covering the whole composition range were prepared, while, for DMPC + DPPC, a step of 0.2 was chosen. The quantities of lipids to reach the desired mixture concentrations were determined gravimetrically using a Sartorius balance yielding a maximal mole fraction uncertainty of \pm 0.002 .

\subsection{Peltier-Element-Based Adiabatic Scanning Calorime-} try ( $p A S C$ ). The working principle of adiabatic scanning calorimetry resides in heating a sample by applying a constant power and monitoring the temperature change over time while maintaining an adiabatic environment [24]. The adiabatic conditions are achieved by minimizing the temperature differences between the sample and the adiabatic shield in order to avoid heat exchange. A major advantage of this technique is that it enables the simultaneous measurement of the temperature dependence of the enthalpy $H$ and of the isobaric heat capacity $C_{p}$. During the experiment the sample temperature $T(t)$ is recorded as a function of time $t$, and the heat capacity $C_{p}(T)$ is calculated via the ratio of the known constant power $P$ and the temperature change $\dot{T} \equiv \mathrm{d} T / \mathrm{d} t$, the latter calculated by numerically differentiating the $T(t)$ data:

$$
C_{p}=\frac{\mathrm{d} Q}{\mathrm{~d} T}=\frac{P \mathrm{~d} t}{\mathrm{~d} T}=\frac{P}{\dot{T}} .
$$


By inverting the measured $T(t)$ data one obtains the enthalpy $H$ :

$$
\begin{aligned}
H & =H_{0}\left(T_{0}\right)+\int_{T_{0}}^{T} C_{p} \mathrm{~d} T=H_{0}\left(T_{0}\right)+\int_{T_{0}}^{T} \frac{P \mathrm{~d} t}{\mathrm{~d} T} \mathrm{~d} T \\
& =H_{0}\left(T_{0}\right)+P\left[t(T)-t_{0}(T)\right],
\end{aligned}
$$

with $T_{0}$ being the starting temperature at the beginning of the run at time $t\left(T_{0}\right)$. The integration can be solved because $P$ is constant in ASC; hence no integration of $C_{p}$ is needed to calculate $H$. The heat capacity of the sample inside the cell was calculated by subtracting the heat capacity of the calorimeter addenda and sample holder (determined from a separate calibration experiment) from the total $C_{p}$. The concept of an ASC experiment is summarized in Figure 1 for the case of a fairly weak transition with considerable pretransitional changes in the heat capacity.

Applying constant power to the sample and measuring the variable rate, as is done in pASC, has a number of interesting consequences towards the behaviour of the sample. The sample is not undergoing a forced temperature rate, like in any DSC-type technique, but is given an amount of energy that is either used for increasing the temperature $T$ of the sample or for the phase conversion (at a constant temperature for a pure substance); the calorimeter follows the behaviour of the sample. Thus, the sample is free to use the heat and controls the calorimeter; the sample remains in thermal and thermodynamic equilibrium. Also, (1) shows that, for a fixed power, the rate and the heat capacity are inversely proportional. As the (effective) heat capacity in the vicinity of a phase transition increases, this means that the rate decreases, and hence the resolution of the experiment increases. Both of these are a marked difference with the DSC-type techniques, including the microcalorimetry that is often used for biological solutions. In these techniques, the sample is forcibly heated at a fixed rate. As a phase transition takes places at a given temperature, the transition heat must be supplied immediately at this temperature, which is physically impossible. The sample is driven out of equilibrium, and the curves are deformed according to the specifics of the instrument and the experiment. This effect is inherent in any constant-rate calorimetric technique, and leads to the apparent rate-dependence of transitions. A more extensive discussion of the advantages of ASC over constant-rate techniques for the high-resolution study of phase transitions is made in [34]. We will also see how this applies to experiments performed on lipid suspensions in Section 3.1.

Practical implementation of the pASC principle requires the power to the sample to be completely known. This is achieved by measuring the applied electrical power with high accuracy, while making sure that only this power is used to heat the sample: all heat leaks from or to the sample have to be eliminated. Therefore the sample is surrounded by a shield that exactly follows the temperature of the sample, so that no heat exchange can take place. This construction can be made such that an absolute accuracy of $2 \%$ is attained for samples of tens of $\mathrm{mg}$, but a much higher resolution is achieved [35].
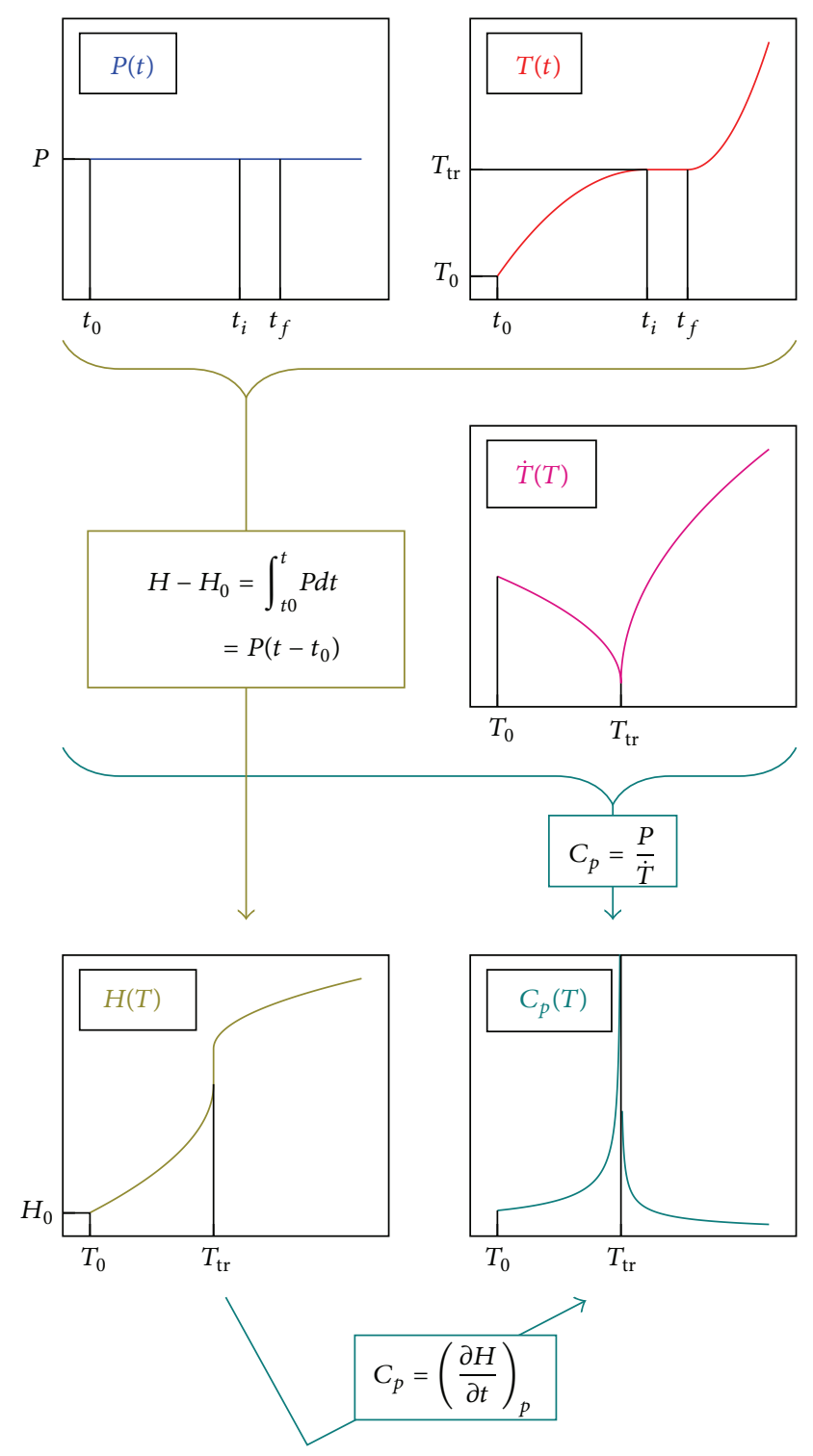

FIGURE 1: Schematic representation of a pASC experiment for a transition with a small latent heat and with anomalous pretransitional enthalpy and heat capacity changes. Starting from the constant applied power $P(t)$ and the measured temperature response of the sample $T(t)$, the enthalpy $H(T)$ and the heat capacity $C_{p}(T)$ can be calculated independently. $T_{0}$ is the starting temperature of the run at the time $t_{0} . t_{i}$ and $t_{f}$ are the beginning and the end of a first-order transition at $T_{\mathrm{tr}}$.

In the pASC setup used for these experiments, schematically depicted in Figure 2, a small sample holder of around $100 \mu \mathrm{L}$ is placed in good thermal contact on the top plate of a very sensitive, semiconductor-based Peltier-element. A small heating element and a miniature thermistor as thermometer are also attached to the top plate. The electrical current provided to the heater of the first shield is controlled by a PID system, which reads the output of the Peltier element, to ensure the required zero (for heating runs) or nonzero (for cooling runs) temperature difference between cell and first shield. The first shield surrounding the sample 


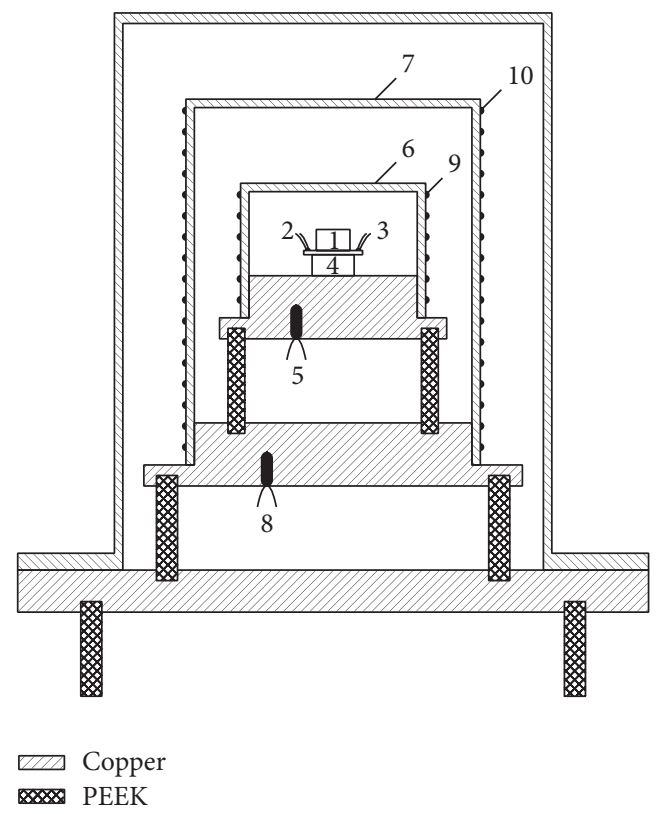

FIGURE 2: Schematic diagram of the Peltier-element-based adiabatic scanning calorimeter. The sample cell 1 is in good thermal contact with the miniature heater 2 and temperature sensor 3 on top of the Peltier element 4 positioned on the bottom part of the first adiabatic shield 6, itself surrounded by another thermal shield 7.9 and 10 are resistive heaters and 5 and 8 resistance thermometers. The whole calorimeter is placed in a temperature controlled oven.

is itself surrounded by an additional shield and an outer can. The second shield follows the temperature of the first shield at a $\sim 0.2 \mathrm{~K}$ lower temperature by means of a PID control system. A Pt thermometer on the second shield is used for controlling the required electric current to be delivered to the heater of the second shield. The calorimeter is placed in a temperature-controlled air chamber that can operate between $-60^{\circ} \mathrm{C}$ and $150^{\circ} \mathrm{C}$ with a stability of $0.1 \mathrm{~K}$. Its temperature can be computer-controlled via a GPIB interface. Heat losses are minimized by evacuating the inside of the calorimeter. By using these different temperature controlled stages, temperature fluctuations at the sample holder are reduced to the $\mu \mathrm{K}$ level. For further details on the structure and functioning of the pASC setup we refer to $[34,35]$.

2.3. Quartz Crystal Microbalance with Dissipation. Quartz crystal microbalance with dissipation monitoring (QCMD) is an acoustic surface-sensitive technique based on the inverse piezoelectric effect. The application of an AC voltage over the sensor electrodes causes the piezoelectric quartz crystal to oscillate at its acoustic resonance frequency. As a result, a transverse acoustic wave propagates across the crystal, reflecting back into the crystal at the surface. When the $\mathrm{AC}$ voltage is turned off, the oscillation amplitude decays exponentially, this decay is recorded, and the frequency $(f)$ and the energy dissipation factor $(D)$ of different overtones are extracted [36]. The dissipation $D$ is the ratio between the dissipated energy during one vibration cycle and the total kinetic and potential energy of the crystal at that moment.
When molecules adsorb to an oscillating quartz crystal, water (or buffer) couples to the adsorbed material as an additional dynamic mass via direct hydration and/or entrapment within the adsorbed film. Thus, the layer is sensed as a viscoelastic hydrogel composed of the molecules and the coupled water together. In our case, it was especially useful to monitor the formation of an intact vesicle layer, since it senses the water coupled to the adsorbed layer.

For the current measurements, we have used QCM-D on a Q-Sense E4 instrument (Q-Sense, Biolin Scientific, Stockholm, Sweden) monitoring the frequency shift $\Delta f$ and the dissipation change $\Delta D$. The Q-Sense $\mathrm{E} 4$ also enables heating or cooling temperature scans from $15^{\circ} \mathrm{C}$ to $50^{\circ} \mathrm{C}$. AT-cut quartz crystals with Au coating (diameter $14 \mathrm{~mm}$, thickness $0.3 \mathrm{~mm}$, surface roughness $3 \mathrm{~nm}$, and resonant frequency $4.95 \mathrm{MHz}$ ) were used. The Au-coated quartz sensors were cleaned with a 5:1:1 mixture of Milli-Q water (conductivity of $0.055 \mathrm{~S} \mathrm{~cm}^{-1}$ at $25^{\circ} \mathrm{C}$ ), ammonia, and hydrogen peroxide and were UV-ozone treated for $15 \mathrm{~min}$ with a Digital PSD series UV-ozone system from Novascan, followed by rinsing in water and drying with $\mathrm{N}_{2}$. The changes in $\Delta f$ and in $\Delta D$ were monitored at five different odd overtones (from 3rd to 11th, the fundamental frequency is rather unstable since this is the one that reaches the farthest out to the edge of the sensor and may be disturbed by the o-ring). First, a baseline with pure HEPES buffer was established and afterwards lipid vesicles were injected over the sensor chip with a flow rate of $50 \mu \mathrm{L} \mathrm{min}^{-1}$. After $10 \mathrm{~min}$ the pump was switched off. Subsequent temperature scans with alternating heating and cooling were performed at a rate of $0.1 \mathrm{~K} \mathrm{~min}^{-1}$, maintaining $30 \mathrm{~min}$ of stabilization between successive ramps. The temperature stability at constant temperature was $\pm 0.02 \mathrm{~K}$.

\section{Results and Discussion}

3.1. Validation of pASC for Lipid Vesicle Calorimetry. Since pASC was only applied before to one sample of a lipid suspension in [21], we decided first to execute some additional validation tests for systematically reduced amounts of DPPC lipid vesicles in water solutions. Starting from an initial solution of $10 \mathrm{mg} \mathrm{mL}^{-1}$, three dilutions were made and measured by pASC with an applied constant power of $180 \mu \mathrm{W}$. This resulted in an average scanning rate, outside the transition region, of about $0.02 \mathrm{~K} \mathrm{~min}^{-1}$. During the transition the temperature rate is substantially reduced, the basic advantage of pASC.

The gradual dilution shows that, even for an absolute amount of $20 \mu \mathrm{g}$ DPPC in the sample, the main transition peak (ripple to liquid-disordered phase) at about $41^{\circ} \mathrm{C}$ remains visible. The gel to ripple phase transition, on the other hand, becomes virtually invisible, a consequence of the much smaller amount of heat involved and its more diffuse character. Focusing on the main transition peak, one clearly observes that although the height of the peak decreases, the onset and end temperature of the peak do not change, indicating that the peak does not diminish due to decreasing sensitivity of the PASC instrument but only due to the decreasing amount of sample and thus decreasing amount 


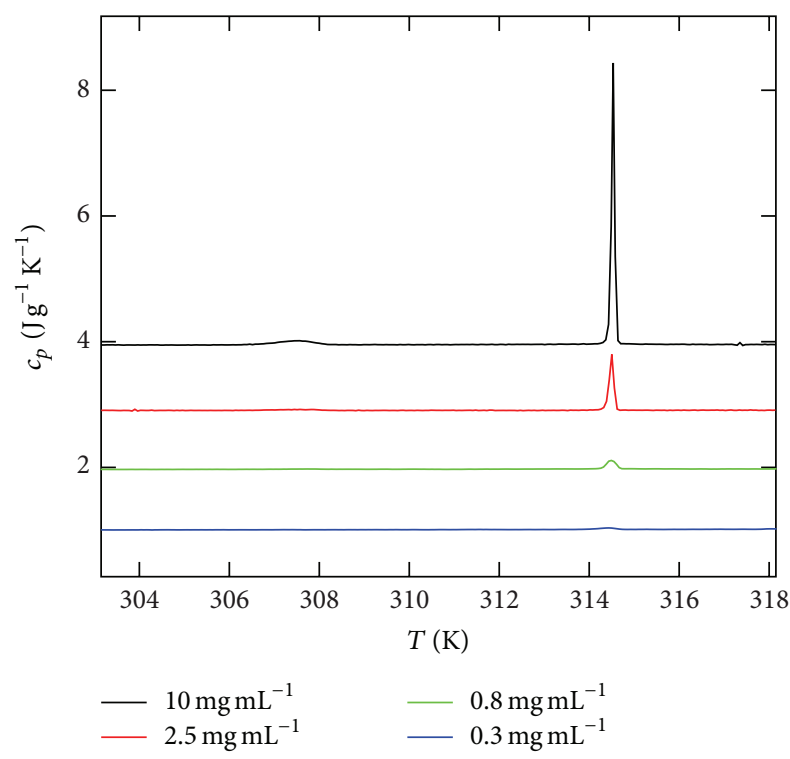

(a)

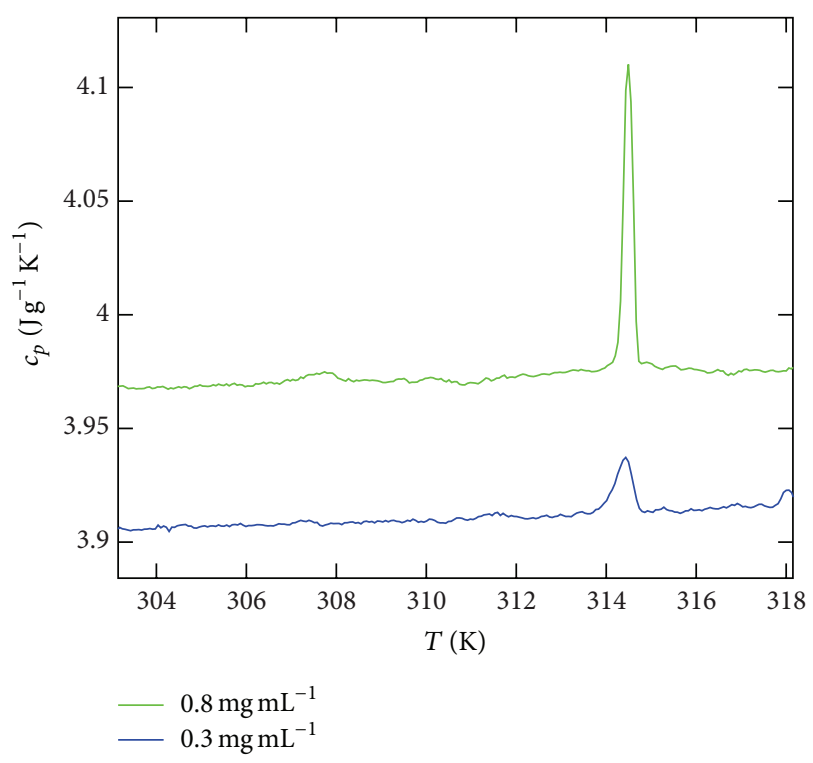

(b)

FIgURE 3: (a) Specific heat capacity $c_{p}(T)$ of a $10 \mathrm{mg} \mathrm{mL}^{-1}$ DPPC vesicle suspension and of three subsequent dilutions as measured with pASC. The curves have been shifted vertically for display reasons and show the total suspension $c_{p}$, not that of the lipid alone. (b) An enlarged view on the most diluted samples.

of transition heat. In fact, when a quantitative analysis of the specific transition enthalpy is made, the results extracted from each of the four runs coincide with each other and are in line with literature data.

The data in Figure 3 illustrate the capability of pASC to perform high-resolution (in temperature as well as in heat capacity) measurements for extremely small amounts of a phase changing compound. Note that the width of the peaks is not related to the instrument, because of applied power independent, but is sample dependent and mainly resulting from the unavoidable presence of impurities in the used lipid.

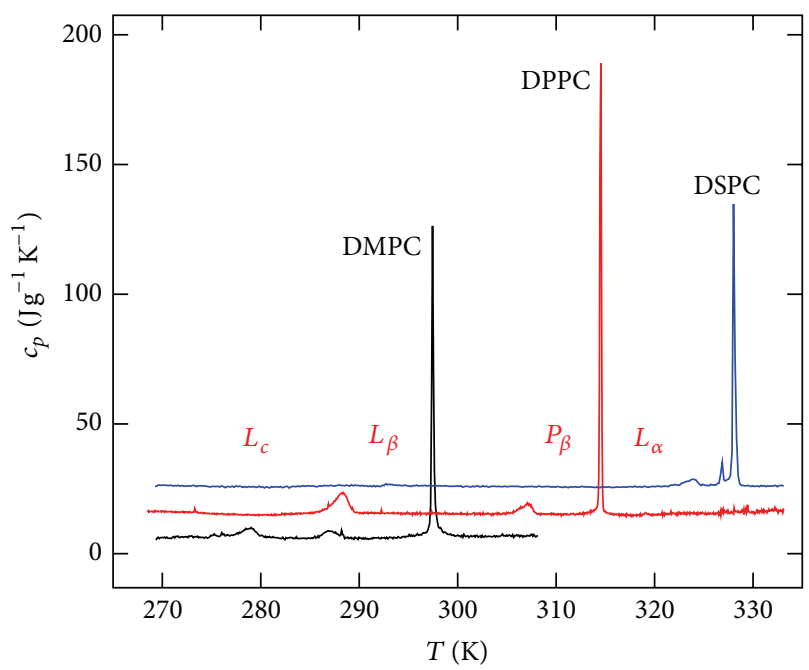

(a)

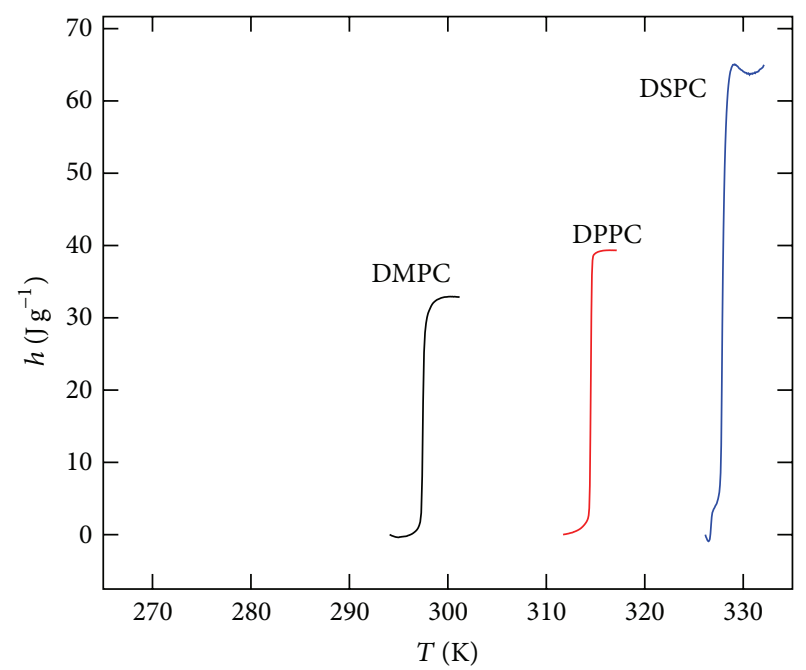

(b)

FIGURE 4: (a) Specific heat capacity $c_{p}(T)$ of the lipids in $10 \mathrm{mg} \mathrm{mL}^{-1}$ dispersions of DMPC (black), DPPC (red), and DSPC (blue). As an example, labels $L_{c}, L_{\beta}, P_{\beta}$, and $L_{\alpha}$ for the different phases are included for DPPC. The $c_{p}$ values for the two last lipids have been shifted upwards for better figure display. (b) $h(T)$ discontinuities at the main phase transition temperature.

\subsection{Peltier-Element-Based Adiabatic Scanning Calorimetry.} The effective specific heat capacity $c_{p}$ of the pure lipids and lipid mixtures was calculated by using $c_{p}$ (sample $)=(1-$ $w) c_{p}$ (water) $+w c_{p}$ (lipid), where $w$ is the weight fraction of the lipid in the buffer solution. Literature $c_{p}(T)$ values of water were used from the International Association for the Properties of Water and Steam (IAPWS) formulation [37].

Figure 4 displays $c_{p}(T)$ and $h(T)$, the latter around the main phase transition temperature, obtained from heating runs with average rates ranging from $\sim 28 \mathrm{mK} \mathrm{min}^{-1}$ to $\sim 44 \mathrm{mK} \mathrm{min}^{-1}$ for the three pure lipids used in this work. The same phase sequence (subgel $L_{\mathrm{c}} \rightarrow$ gel $L_{\beta} \rightarrow$ ripple $P_{\beta} \rightarrow$ liquid-disordered $L_{\alpha}$ ) was encountered for these three lipids and all three phase transitions are clearly first-order, since 


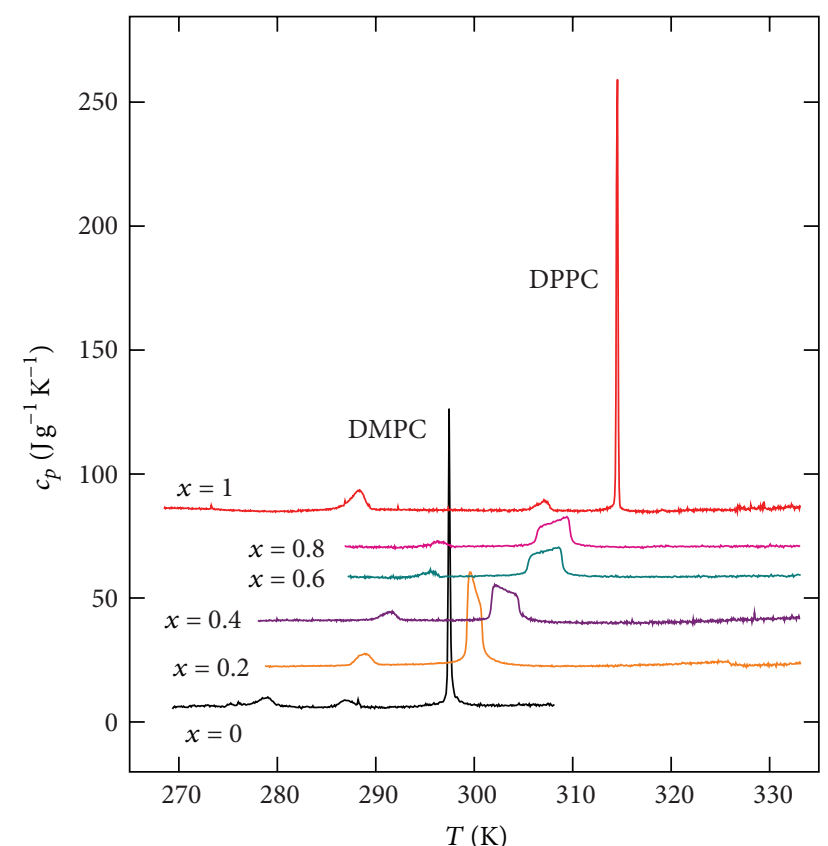

(a)

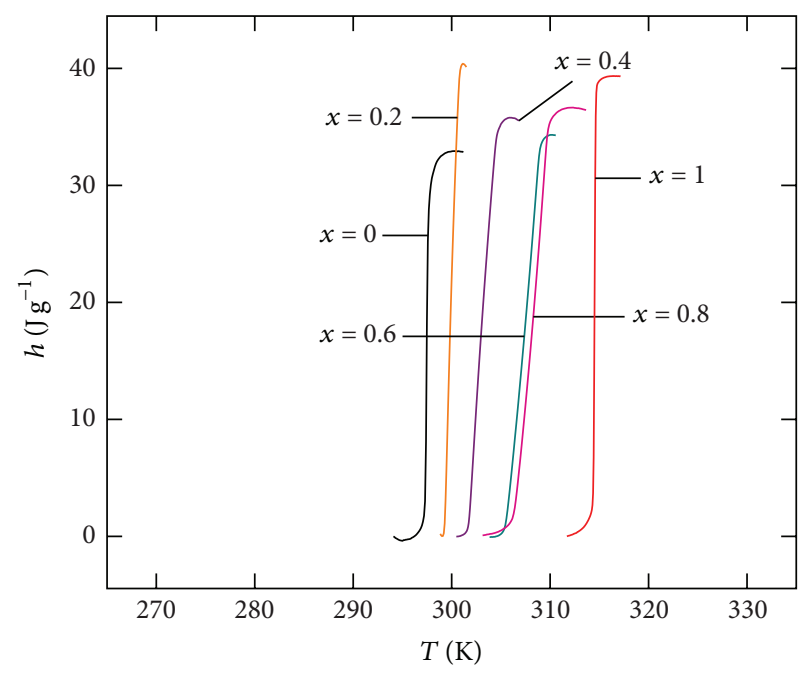

(b)

FIGURE 5: (a) Specific heat capacity $c_{p}(T)$ of the lipids in $10 \mathrm{mg} \mathrm{mL}^{-1}$ dispersions of DMPC + DPPC mixtures over the complete concentration interval. (b) $h(T)$ discontinuities at the main phase transition temperature for the mixtures displayed. The $c_{p}$ values have been shifted for better display; $x$ refers to the mole fraction of DPPC in the lipid mixture.

they exhibit a clear discontinuous jump in the enthalpy (nonzero latent heat). For DSPC, an additional feature can be seen close to the ripple $\rightarrow$ liquid-disordered or "main phase transition," in agreement with previous experimental studies [38]. For the mixtures, Figures 5 and 6 also show that there is a, now broad, main transition, accompanied by transitions at lower temperature. In this part, we focus on the transitions to the liquid-disordered $L_{\alpha}$ phase, while we will discuss the lower temperature transitions in Section 3.4.

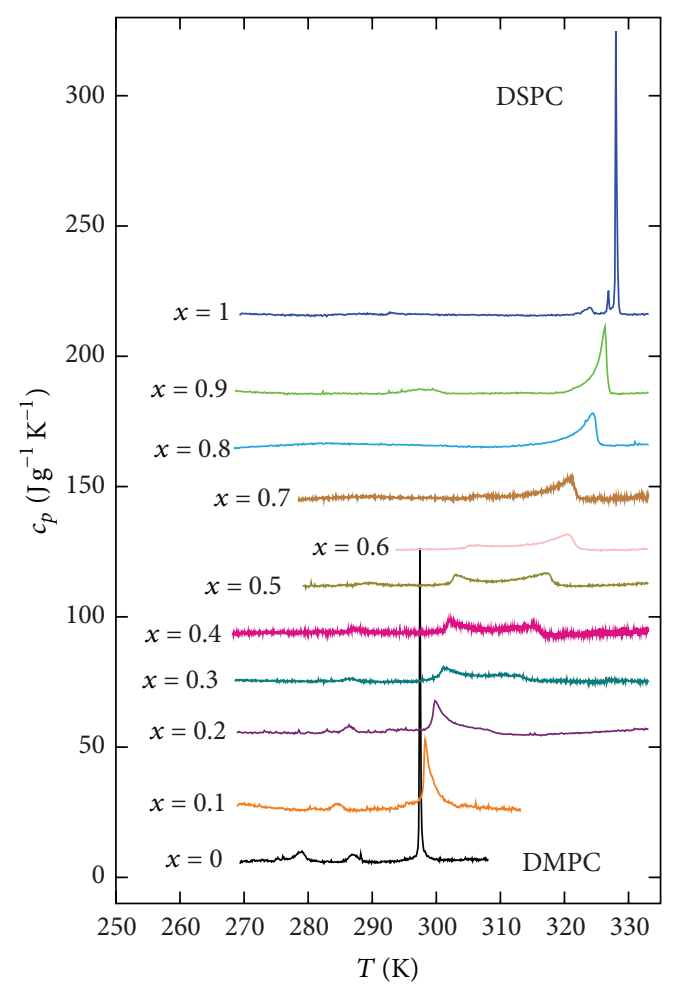

(a)

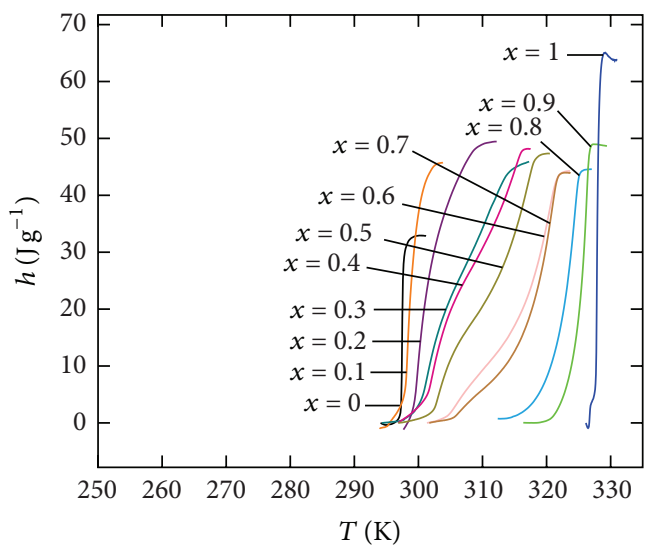

(b)

Figure 6: (a) Specific heat capacity $c_{p}(T)$ of the lipids in $10 \mathrm{mg} \mathrm{mL}$ dispersions of DMPC + DSPC mixtures over the complete concentration interval. (b) $h(T)$ discontinuities at the main phase transition temperature for the mixtures displayed. The $c_{p}$ values have been shifted for better display; $x$ refers to the mole fraction of DSPC in the lipid mixture.

The measurement of $h(T)$ provides a unique tool for determining the total heat of the transition $\Delta h$ and the latent heat $L$. Although often used interchangeably, there is a difference between those two quantities. $\Delta h$ is the total transition heat, the enthalpy difference over the entire jump, and it includes the contribution from the latent heat on the one hand and from contributions of pretransitional fluctuations on the other hand. The latent heat $L$ is the heat needed to 
TABLE 1: Transition temperatures, total heats, latent heats, and cooperativity units of the gel to ripple transition and the ripple to liquiddisordered transition of the three pure phospholipids obtained from pASC measurements.

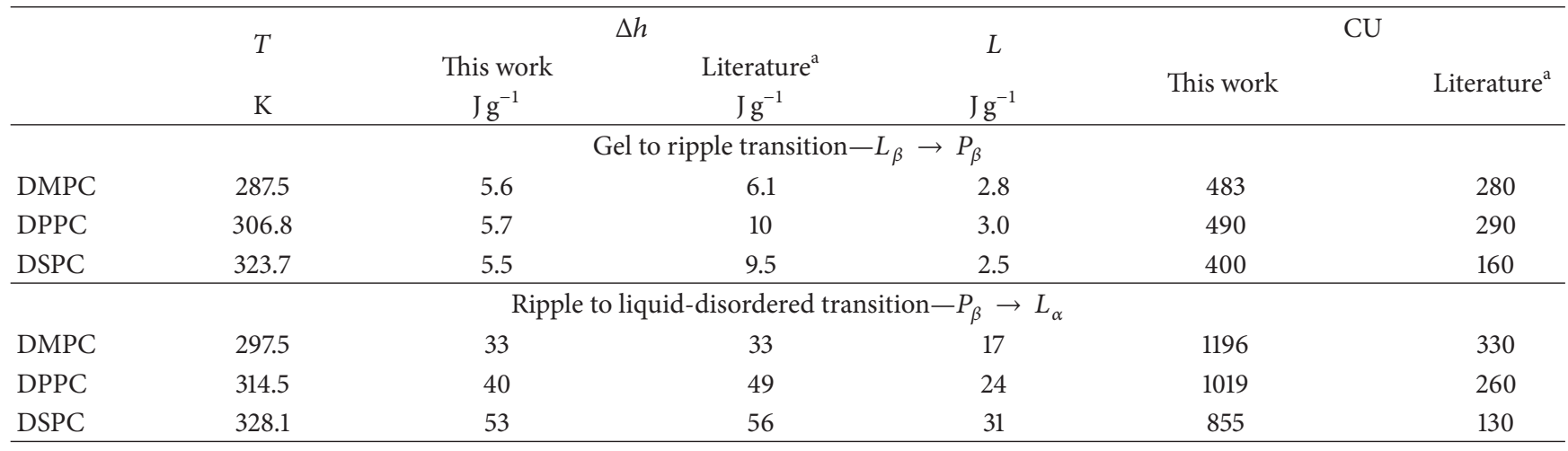

${ }^{\mathrm{a}}$ Literature values are taken from Mabrey and Sturtevant [27].

TABLE 2: Onset and completion temperatures, total heats, and cooperativity unit values for DMPC + DPPC mixtures obtained from pASC measurements, as well as onset and completion temperatures obtained from QCM-D measurements.

\begin{tabular}{|c|c|c|c|c|c|c|}
\hline \multirow{3}{*}{$x_{\mathrm{DPPC}}$} & \multicolumn{2}{|c|}{$T_{\text {solidus }}$} & \multicolumn{2}{|c|}{$T_{\text {liquidus }}$} & \multirow{2}{*}{$\Delta h$} & \multirow{3}{*}{$\mathrm{CU}$} \\
\hline & pASC & QCM & pASC & QCM & & \\
\hline & K & K & K & K & $\mathrm{Jg}^{-1}$ & \\
\hline 0.00 & 297.5 & 297.4 & 297.5 & 297.4 & 33 & 1196 \\
\hline 0.20 & 299.2 & 298.5 & 301.1 & 301.7 & 40 & 116 \\
\hline 0.40 & 301.6 & 300.7 & 304.8 & 304.3 & 36 & 85 \\
\hline 0.60 & 305.1 & 303.7 & 309.2 & 309.0 & 37 & 64 \\
\hline 0.76 & 305.9 & 306.3 & 310.0 & 311.7 & 37 & 72 \\
\hline 1.00 & 314.5 & 314.4 & 314.5 & 314.4 & 40 & 1019 \\
\hline
\end{tabular}

overcome the pure coexistence region and corresponds to the steep linear part of the enthalpy jump. These notions are well developed for liquid-crystalline systems [39, 40], and the same determination methods are used in this work. The transition temperatures, $\Delta h$ and $L$, for the pure lipids are included in Table 1, and all three quantities increase with increasing alkyl chain length, as observed in previous literature studies using DSC [27].

The sharpness of the heat capacity peak of the main phase transition (ripple to liquid-disordered) reveals that it is highly cooperative for all three lipids; namely, a very large fraction of the lipid molecules go through the phase transition simultaneously. A measure of this cooperation between phospholipid molecules in the bilayer is given by the cooperative unit CU. It is defined as the ratio between the van't Hoff enthalpy $\Delta h_{\mathrm{VH}}$ and the calorimetric enthalpy $\Delta h$ of the transition [28]:

$$
\mathrm{CU}=\frac{\Delta h_{\mathrm{VH}}}{\Delta h}
$$

A good estimate for CU can be obtained by combining the experimental $\Delta h$ from pASC with the following approximation for $\Delta h_{\mathrm{VH}}[28]$ :

$$
\Delta h_{\mathrm{VH}}=\frac{R T_{m}^{2}}{\Delta T_{1 / 2}},
$$

where $R$ is the gas constant, $R=8.3144 \mathrm{~J} \mathrm{~mol}^{-1} \mathrm{~K}^{-1}, T_{m}$ is the temperature at the maximum of the $c_{p}(T)$ curve, and $\Delta T_{1 / 2}$ is the temperature width at half height of the $c_{p}(T)$ curve. In Table 1 it can be observed that, within mutual resolution, there is consistency between the DSC literature and our new pASC values for the total $\Delta h$ values of the pure compounds. However, literature values for CU are systematically lower than ours. We believe this is the result of a systematic overestimation of the width $\Delta T_{1 / 2}$ by unavoidable peak broadening in DSC measurements [34]. The degree of cooperativity slightly decreases with the length of the alkyl chain as can be seen from the cooperativity unit values included in Table 1.

The same type of analysis has also been applied to the mixtures. However, in contrast to the sharp main transition in the pure lipids, this transition is much wider in the mixtures, and only the total transition heat $\Delta h$ can be determined; the concept of a latent heat $L$ cannot be established for these transitions. Tables 2 and 3 summarize the relevant information concerning mixtures of DMPC + DPPC and DMPC + DSPC, respectively, while Figures 5 and 6 show $c_{p}(T)$ and $h(T)$ of the studied mixtures.

At the melting or main phase transition, DMPC + DPPC mixtures exhibit a clear phase coexistence region, and the single $c_{p}$ peak characteristic of the main phase transition is shifted towards higher temperatures and broadens upon addition of DPPC. Moreover, it displays a marked shape evolution: a second feature appears, gaining height when 
TABLE 3: Onset and completion temperatures, total heats, and cooperativity units for DMPC + DSPC mixtures obtained from pASC measurements, as well as onset and completion temperatures obtained from QCM-D measurements.

\begin{tabular}{|c|c|c|c|c|c|c|}
\hline & & & & & $\Delta h$ & \\
\hline$x_{\mathrm{DPPC}}$ & pASC & QCM & pASC & QCM & & $\mathrm{CU}$ \\
\hline & $\mathrm{K}$ & $\mathrm{K}$ & K & K & $\mathrm{Jg}^{-1}$ & \\
\hline 0.0 & 297.5 & 297.4 & 297.5 & 297.4 & 33 & 1196 \\
\hline 0.1 & 297.8 & 297.8 & 299.8 & 301.7 & 43 & 51 \\
\hline 0.2 & 299.2 & 298.8 & 310.7 & 308.1 & 47 & 19 \\
\hline 0.3 & 299.7 & 299.8 & 315.5 & 312.8 & 47 & 15 \\
\hline 0.4 & 301.2 & 301.7 & 316.4 & 316.0 & 47 & 12 \\
\hline 0.5 & 301.9 & 302.5 & 318.5 & 318.9 & 47 & 11 \\
\hline 0.6 & 303.7 & 303.6 & 322.3 & 320.0 & 43 & 12 \\
\hline 0.7 & 307.6 & 307.2 & 322.6 & $-^{a}$ & 43 & 13 \\
\hline 0.8 & 320.4 & 317.5 & 325.2 & $-^{a}$ & 43 & 18 \\
\hline 0.9 & 323.7 & 315.4 & 326.8 & $-^{a}$ & 48 & 28 \\
\hline 1.0 & 328.1 & $-^{\mathrm{a}}$ & 328.1 & $-^{a}$ & 53 & 855 \\
\hline
\end{tabular}

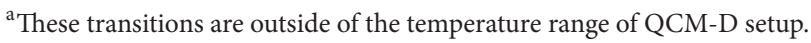

increasing the amount of DPPC. For DMPC + DSPC mixtures, the phase coexistence region is delimited by two welldefined peaks, departing more from ideality due to the higher dissimilarity (larger difference in alkyl chain length) between DMPC and DSPC. The second peak increases in height with increasing the amount of DSPC, while the first one vanishes as one approaches pure DSPC. As expected, at the equimolar mixture the two peaks display the same height. Figures 5(b) and 6(b) show the evolution of $h(T)$ for both types of mixtures. Within the estimated uncertainty, the enthalpy jumps evolve from smaller to higher values upon the addition of the second (longer chain) phospholipid component. Moreover, the slope of the enthalpy jump decreases as the mixtures approach equimolarity. An equivalent effect is reflected in Figure 7, which displays $\Delta h$ and CU values.

The curves of concentrations in between two pure compounds reveal combined effects of the two respective individual gels to liquid-disordered phase transitions, whose contribution scales with the concentration of the respective compound. That one does not observe two separate peaks in $c_{p}$ or separate steps in the specific enthalpy curves at the respective transition temperatures of the pure compounds is a clear indication that one does not have a system with two types of single-component vesicles, but rather with vesicles with both types of lipids present in the membranes (roughly in proportions to respective the lipid mole fractions). The fact that in the mixtures the beginning and the end of the transition region deviate from the melting temperatures of the pure compounds indicates that the melting of the chains of each type of lipid is strongly affected by the presence of the other type in the membrane. The quite low values of the $\mathrm{CU}$ in the mixtures point to quite small (but larger than single molecules) units (of different sizes) in the membranes in comparison with the pure membranes.

Indeed, the calculated $\mathrm{CU}$ for the different types of mixtures nicely evidences the gradual loss of cooperativity as the mixtures approach the equimolar value. Noteworthy, for the same concentrations of the second component, the degree of cooperativity is higher in DMPC + DPPC mixtures than in DMPC + DSPC ones, an additional evidence of the nonideal behaviour of DMPC + DSPC mixtures. Due to the high values of the CU of the pure lipids ( 1000), they have not been included in the plot for clarity reasons.

\subsection{Quartz Crystal Microbalance with Dissipation Monitoring.} Vesicle deposition was carried out at $16^{\circ} \mathrm{C}$ for all prepared mixtures and the behaviour of both $\Delta f / n$ and $\Delta D$ was monitored. This temperature was chosen to stabilize the vesicles and to obtain an intact SLV [41]. Figure 8 illustrates the process of vesicle adsorption for a representative mixture, namely, DMPC + 0.1 DSPC. The same pattern of behaviour was obtained for the remaining mixtures. As can be observed in Figure 8, the overtones do not overlap and the responses in both frequency and dissipation are large. One can thus conclude that the adsorbed vesicle layer is a viscoelastic layer. Therefore, since the layer is not rigid, it is not possible to use the Sauerbrey relation and a Kelvin-Voigt based model is needed to obtain information about the viscoelastic properties of the layer. The model basically consists of a spring in parallel with a dashpot, thus combining both the elastic and the viscous characters of the film [42].

Heating and cooling cycles were performed after vesicle adsorption to assess the changes in frequency and dissipation, comparing the sensors with vesicles adsorbed and the sensors with only the HEPES buffer solution, for the pure lipids and also for the binary mixtures. As an example, Figure 9 shows the results for the DMPC +0.1 DSPC mixture. The sensor only exposed to HEPES buffer (reference sensor) displays a regular (almost linear) behaviour (see Figure 9(b)). The frequency shift increases with increasing temperature and dissipation change, in turn, exhibits an opposite behaviour [20, 21]. Such regular behaviour is lost when the vesicles are adsorbed: anomalies in both dissipation and frequency are observed for pure phospholipids and for the mixtures, revealing that structural changes are taking place and constituting a clear 


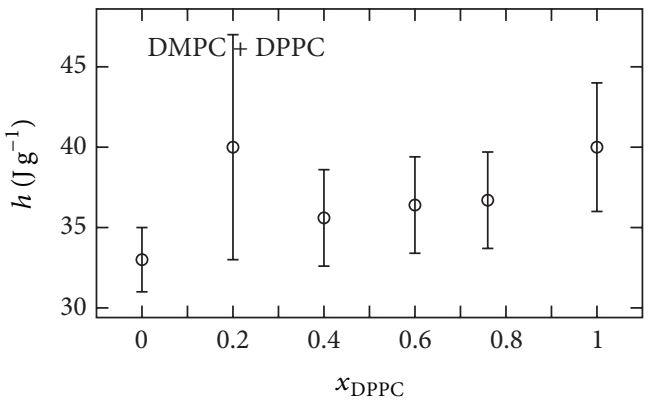

(a)

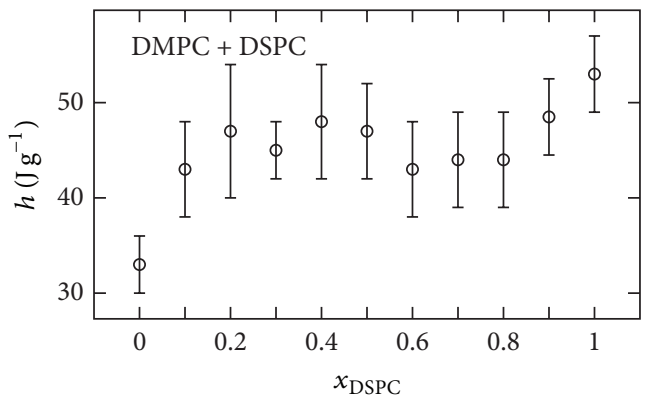

(c)

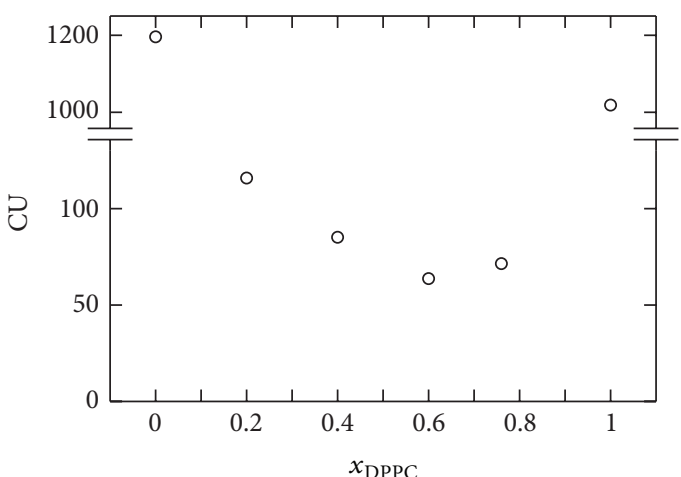

(b)

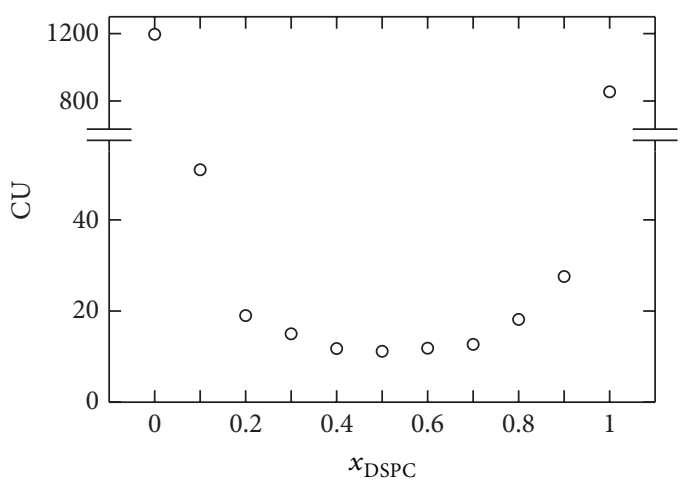

(d)

FIgURE 7: Transition heat and CU (Equations (3) and (4)) results for the lipid mixtures. (a) Total heat $\Delta h$ and (b) cooperative unit CU for DMPC + DPPC; (c) $\Delta h$ and (d) CU for DMPC + DSPC.

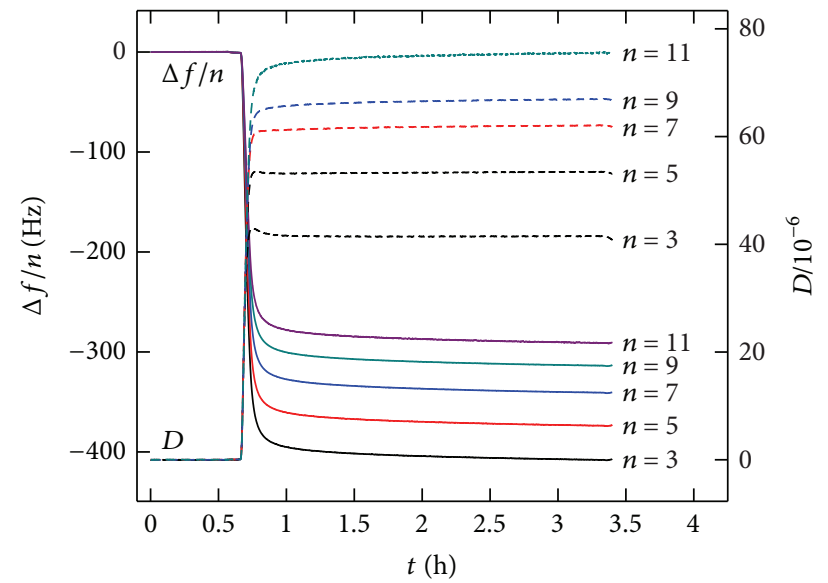

FIGURE 8: Time evolution of $\Delta f / n$ (solid lines) and $\Delta D$ (dashed lines) during a typical QCM-D experiment of vesicle adsorption on gold-coated quartz at $16^{\circ} \mathrm{C}$. Data for the DMPC + 0.1 DSPC mixture.

signature of phase transitions. Figure 9(c) shows normalized frequency and dissipation values, obtained by substrating the HEPES (reference) response to the response of the sensors containing vesicles.

The effective viscosity $\eta$ was calculated using the KelvinVoigt-based model. The data of several overtones $(n=3$ to $n=11$ ) were fitted using the software Qtools (Q-Sense, Biolin
Scientific, Stockholm, Sweden) keeping as fixed parameters the density of the lipid layer $\left(1.06 \mathrm{~g} \mathrm{~cm}^{-3}\right)$ [43], the density of the fluid $\left(1.0 \mathrm{~g} \mathrm{~cm}^{-3}\right)$, and the viscosity of the fluid (1 mPa s). Figures 10 and 11 display the temperature dependence of the viscosity for mixtures of DMPC + DPPC and DMPC + DSPC, respectively. Upon cooling, the viscosity responses show a similar shape as upon heating with $\sim 1.5 \mathrm{~K}$ hysteresis. For all the mixtures, the change from the gel state to the liquiddisordered state is reflected in the viscosity behaviour, which decreases from a more viscous state (gel phase) to a less viscous one (liquid-disordered). A similar kind of temperature profile for the viscosity was reported for DPPC + cholesterol vesicle dispersions using a laser-induced ultrasonic probe [13]. In our case, viscosity values should be taken as effective and not as absolute values because the model assumes a homogeneous vesicle layer and our vesicle dispersion was not strictly monodisperse. At a given temperature, where phase separation starts, a sudden decrease in $\eta$ is observed, a fraction of DMPC lipids starts to change phase, and the layer is in a coexistence region between gel and liquiddisordered phases. Once all the lipids have completed the phase conversion, a regular behaviour is recovered since all lipids are in the liquid-disordered phase.

For DMPC + DPPC mixtures, the slope of the changes in $\eta$ during phase coexistence decreases as the mixtures approach equimolarity, indicating that the width of the gel + liquid-disordered coexistence region is larger. This is 


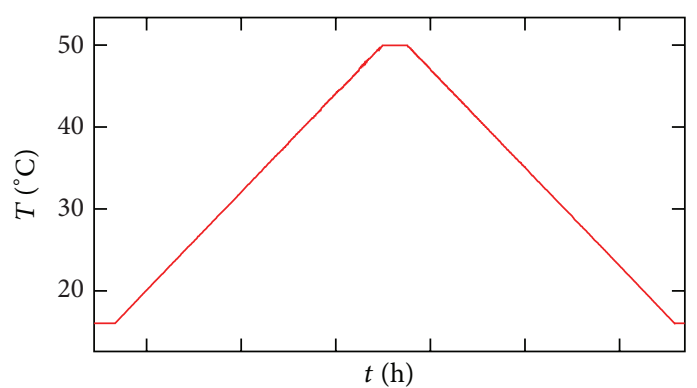

(a)

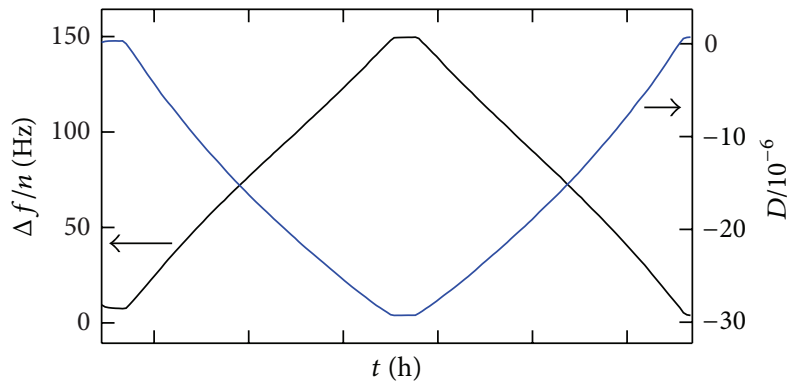

(b)

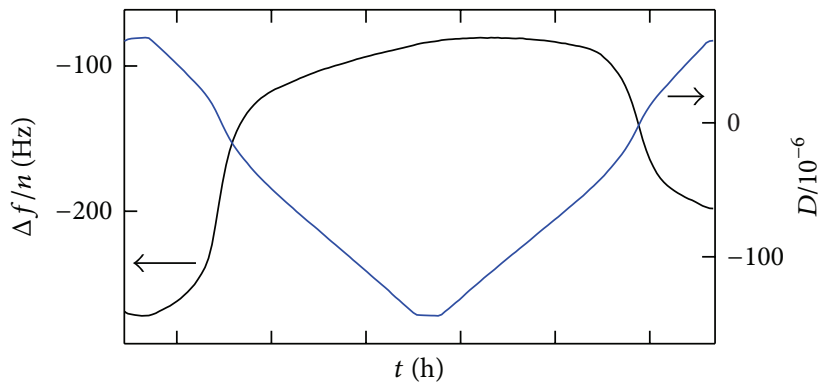

(c)

FIGURE 9: (a) Time dependence of the sensor temperature during the experiments. (b) $\Delta f / n$ (black) and $\Delta D$ (blue) of the $n=9$ overtone for a blank QCM-D sensor with HEPES buffer. (c) $\Delta f / n$ (black) and $\Delta D$ (blue) of the $n=9$ overtone for the sensor with HEPES buffer and vesicles adsorbed; data for the DMPC + 0.1 DSPC mixture.

a direct consequence of the loss of cooperativity during the transition caused by the increasing amount of a different lipid component and the size of this width increases with the amount of DPPC. The DMPC + DSPC mixtures follow the same pattern of behaviour, showing an increase of the effective value of viscosity for concentrations $x_{\mathrm{DSPC}} \geq 0.5$. At concentrations around the equimolar composition $\left(x_{\mathrm{DSPC}}=\right.$ 0.3 to 0.7 ), an intermediate feature (change in slope) can be observed. For mixtures with higher concentration of DSPC $(x=0.8,0.9$, and 1$)$, the high temperature transition at the liquidus line is at temperatures above the upper instrumental limit of the QCM-D (maximum $T=50^{\circ} \mathrm{C}$ ).

Finally, let us note the similarity between the $h(T)$ and the $\eta(T)$ data. In both data sets, the transitions are visible as sudden changes in the respective properties, which for the pure systems take essentially the shape of a step. Of course, $h(T)$ is increasing with temperature, whereas $\eta(T)$

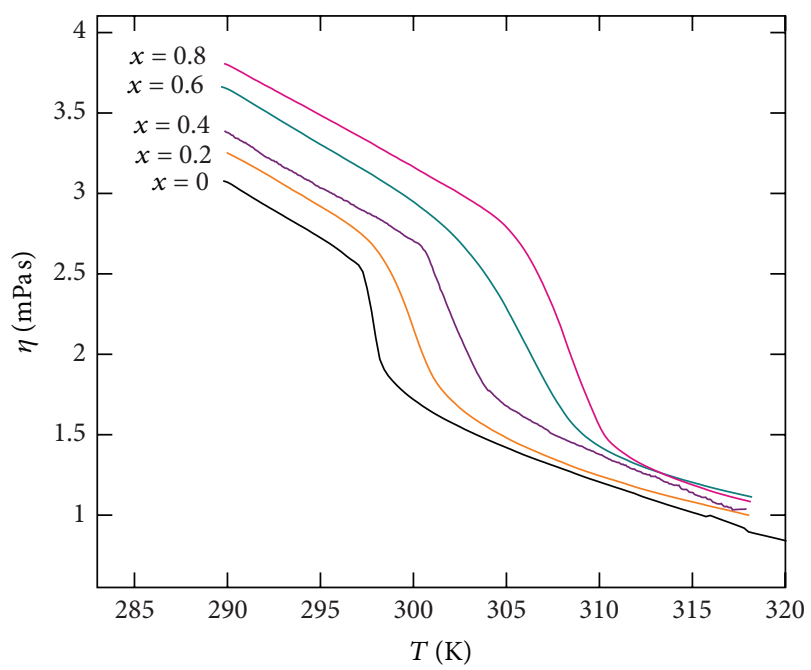

FIgURE 10: Temperature dependence of the calculated effective viscosity $\eta$ of a supported vesicle layer of DMPC + DPPC mixtures on a gold-coated QCM-D quartz sensor. $x$ refers to the mole fraction of DPPC.

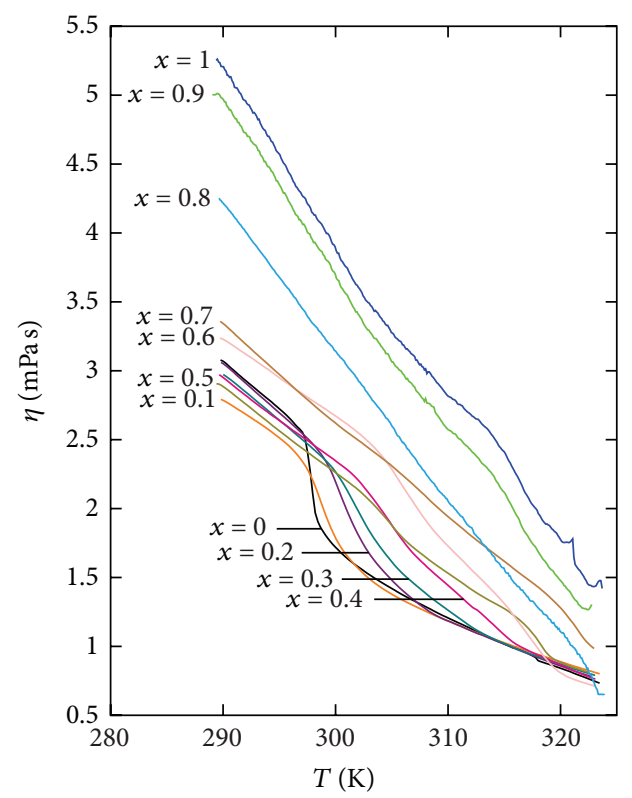

Figure 11: Temperature dependence of the calculated effective viscosity $\eta$ of a supported vesicle layer of DMPC + DSPC mixtures on a gold-coated QCM-D quartz sensor. $x$ refers to the mole fraction of DSPC.

is decreasing, but this does not influence the potential for analysis: the position and width of step can be used to determine the phase transition properties. The exact determination of the phase transitions temperatures requires in both cases similar constructs, namely, the tangent method [33]. However, in the case of thermal data, the presence of peaks in $c_{p}(T)$, which is the temperature derivative of $h(T)$, provides a more convenient method for the determination of transition temperatures. 


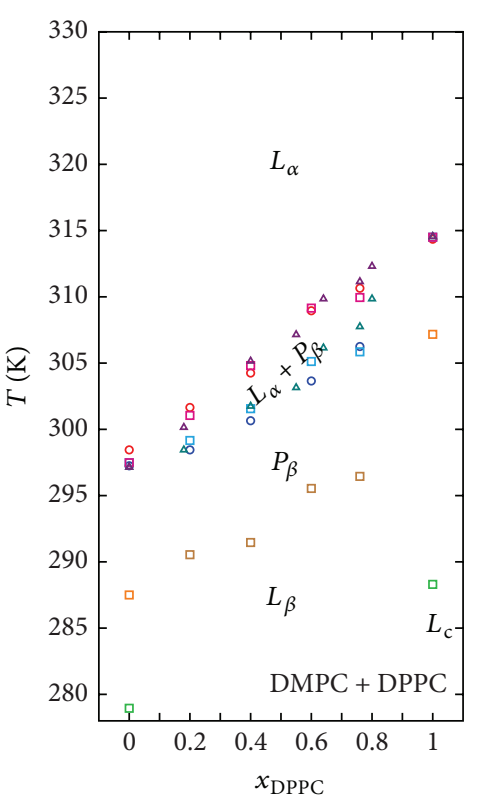

(a)

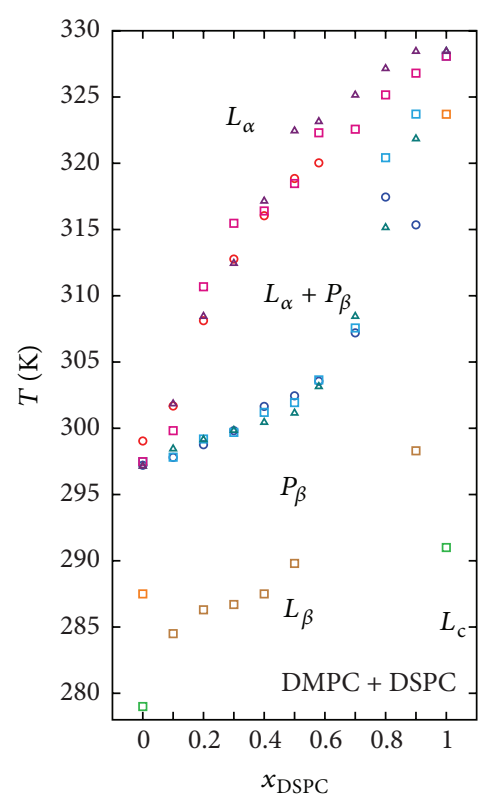

(b)

FIGURE 12: Phase diagram of (a) DMPC + DPPC mixtures and (b) DMPC + DSPC mixtures. Circles denote the values obtained with QCM$\mathrm{D}$, squares are pASC data, and triangles are literature values [27]. Reddish colours indicate the upper limit of the phase coexistence, blueish colours the lower limit. Green and brownish symbols denote the low-temperature transitions: the brownish symbols indicate the transition from the ripple phase to a lower-temperature phase and the green symbols the gel to sub-gel transition in the pure lipids.

3.4. Low-Temperature Transitions. The discussion up till now has been focused on the main transition region of these mixtures. But pASC has also detected lower-temperature transitions, in the temperature regions corresponding to those of the gel to ripple transition. On the other hand, the viscosity as measured by QCM-D appears to be an insufficiently sensitive probe for these transitions. In this section, they will be shortly discussed, before we bring all information together in the phase diagrams in the next section.

The low-temperature phases of pure phosphatidylcholines are quite well studied [44], and, in addition to the prototypical $L_{c} \rightarrow L_{\beta} \rightarrow P_{\beta} \rightarrow L_{\alpha}$ phase sequence for the three lipids in this work, extra complexity is present in the form of intermediate phases and metastable states [44, 45]. On the other hand, literature data for these mixtures seem much more scarce.

First, we note that in the studied temperature range (down to about $280 \mathrm{~K}$ for the mixtures), we only observe a single phase transition below the coexistence region. Thermal data cannot give any definite assignment of the phases, although the properties of the phase transitions may, by analogy, give some indication. From a first glimpse at the phase diagram in Figure 12, it appears that, for both mixtures, the lowtemperature transition follows the behaviour of the gel to ripple transitions in the pure lipids. This observation leads to the potential assignment of the phase above this transition as the ripple phase $P_{\beta}$. This is supported, in the particular case of DMPC + DSPC, by AFM data of a supported double bilayer, of which the top bilayer clearly show the rippled texture, both in the coexistence region and at lower temperature; these authors also report this transition at $288 \mathrm{~K}$ from DSC data [46]. Similar results have also been obtained for DLPC + DSPC [47].

Since we have not observed a transition at lower temperatures in the mixtures, we cannot make any statement about the nature of the lower phase, although it may be assumed that the occurrence of lipids with different lengths favours the somewhat more disordered $L_{\beta}$ gel phase and widens this region at the expense of the ordered crystalline $L_{c}$ subgel phase. The low-temperature transition peaks of the mixtures are not very well resolved and rather broad; in particular, there is nothing reminiscent of a (double-peak) two-phase region. Finally, we note that the line of low-temperatures phase transition in the mixtures more or less has the same behaviour as the lower limit of the main transition, resulting in a similar width for the supposed ripple phase. Thus, our work is consistent with the earlier assessment of these mixtures [48].

3.5. Comparison between QCM-D and pASC Results: Phase Diagram. Finally, the phase transition temperatures are extracted from the pASC and QCM-D data and combined in the phase diagrams. These results are depicted in Figure 12 and the temperatures are included in Tables 1, 2, and 3. For the lower-temperature transitions, single temperature is given. The broad coexistence region is delimited by two temperatures: the onset temperatures of the main transition at each concentration define to so-called solidus line, while the completion temperatures define the liquidus line. The broad temperature range of the observed phase changes 
makes the determination of the beginning and the end of phase coexistence not straightforward. In order to minimize arbitrary choices, we followed a systematic procedure based on an equivalent approach to the one used in previous studies known as the tangent method [33].

For the pASC experiments, the transition temperatures have been obtained from the $c_{p}(T)$ curves, whereas the QCM$\mathrm{D}$ transition temperatures have been extracted from the $\eta(T)$ curves. In both cases, the intersection of the tangent of the regular part of $c_{p}$ and $\eta$ at its low temperature (onset of the phase transition) or high temperature (completion of the phase transition) with the tangent to the jump at the phase transition in both properties yielded the values of the phase boundary temperatures. Because the upper limit of the QCM-D setup is $323.15 \mathrm{~K}$, this determination method yields a higher uncertainty for the temperatures of the highest DSPC concentrations of DMPC + DSPC mixtures.

Figure 12 shows in the first place that there is good agreement between the results obtained by pASC and QCMD. Moreover, our results coincide with the literature data of Mabrey and Sturtevant [27]. For DMPC + DPPC, we find the small coexistence region, showing the greater similarity between the lipids in this mixture than in DMPC + DSPC with its broad and asymmetric coexistence region.

\section{Summary and Conclusions}

In this work, we have shown that Peltier-element-based adiabatic scanning calorimetry and quartz-crystal microbalance with dissipation monitoring can be used to study the phase transitions of lipid vesicles. Both methods lead to comparable conclusions, although pASC measures the vesicles in suspension, whereas QCM-D operates on a deposited vesicle layer on top of the sensor.

At this point, we should mention that there is some influence of the size of the vesicles on the transition temperatures, provided the vesicles are small enough. Biltonen and Lichtenberg have evaluated the influence of size on the melting temperature for bulk systems using differential scanning calorimetry and concluded that, above $70 \mathrm{~nm}$, the melting temperature remains rather constant, while, below $70 \mathrm{~nm}$, it decreases as vesicle size decreases [49]. For the pASC experiments in this work, the vesicles are multilamellar and of micrometer order, while those used in QCM-D are far above $70 \mathrm{~nm}$, so, in principle, size does not influence much the main phase transition temperature in our measurements. As regards substrate influence, it strongly affects supported lipid bilayers, where the melting of the two leaflets is decoupled due to the strong interactions of the lower leaflet with the substrate. But, in this work, with a supported vesicle layer, the influence of the substrate on supported vesicle layers is much smaller than in supported lipid bilayers.

These methods have been applied to carry out a detailed study of phase transitions of binary mixtures of DMPC + DPPC and DMPC + DSPC and of the pure components. Despite the difference in geometry of the studied systems (multilamellar vesicles in aqueous dispersion by pASC and supported vesicle layer on gold by QCM-D), the results obtained are mutually consistent. Phase diagrams were constructed from $c_{p}(T)$ and $\eta(T)$ data, yielding similar results in agreement with previous literature. The approach for determining phase transition temperatures from the analysis of both quantities is equivalent, corroborating the compatibility of both methods.

Apart from the coexistence region, pASC enabled the study of low-temperature transitions, mapping the phase boundary between the gel-type phases for both types of lipid mixtures. These data suggest that the mixtures form a ripple phase below the main transition.

The total heat $\Delta h$ and the degree of cooperativity CU were also determined from pASC measurements. $\Delta h$ exhibits a clear tendency towards higher transition energies with increasing concentration of longer lipid chains. CU exhibits a minimum at the equimolar composition, reflecting the gradual loss of intermolecular cooperation in lipid mixtures during melting. This observation could be consistent with the phase transition behaviour only being influenced by local interactions on spatial scales much shorter than the mescoscopic sizes of vesicles. We also note that the CU are considerably larger as obtained by pASC than in literature [27], obtained by DSC. Although differences in levels of impurity might play a role, the main reason for this difference might be the fundamental difference in operation principle between these two calorimetric techniques (see Section 2.2).

The molecular differences between the components of the studied mixtures (difference in hydrocarbon chain length) are reflected in the measured physical properties as well as in the shape of the obtained phase diagrams. The responses observed are more pronounced in DMPC + DSPC mixtures (difference of four ethyl groups) than in DMPC + DPPC mixtures (difference of two ethyl groups). Heat capacity temperature curves display two well-rounded peaks for DMPC + DSPC, while there is only an emerging feature in DMPC + DPPC. Moreover, enthalpy and viscosity temperature profiles show also an additional feature in DMPC + DSPC mixtures.

In summary, we have shown the capability of these two experimental techniques to map the phase behaviour of pure lipids and lipid mixtures. pASC is highlighted as a powerful tool for getting high-resolution thermodynamic data, while QCM-D is an alternative and simple way to map phase diagrams by effective viscosity measurements, enabling to mimic lipid interfaces with biological relevance. In this respect, this work can be taken as a departure point for further studies on more complex lipid mixtures displaying biologically relevant phases such as the liquid-ordered phase.

\section{Conflict of Interests}

The authors declare that there is no conflict of interests regarding the publication of this paper.

\section{Acknowledgments}

This work was supported by the Belgian Province of Limburg in the framework of "Life-Science Initiative", the Flemish Government in the framework of the Methusalem project NANO Hasselt-Antwerp, the Research Foundation Flanders 
FWO, Projects 6.0B62.13N and G.0492.10, and the Special Research Fund BOF of Hasselt University. The authors thank Professor Marlies Van Bael for authorizing the use of the dynamic light scattering setup.

\section{References}

[1] M. S. Bretscher, "Membrane structure: some general principles," Science, vol. 181, no. 4100, pp. 622-629, 1973.

[2] M. Eeman and M. Deleu, "From biological membranes to biomimetic model membranes," Biotechnologie, Agronomie, Société et Environnement, vol. 14, no. 4, pp. 719-736, 2010, http://popups.ulg.ac.be/1780-4507/index.php?id=6568.

[3] K. Simons and E. Ikonen, "Functional rafts in cell membranes," Nature, vol. 387, no. 6633, pp. 569-572, 1997.

[4] A. Pokorny, L. E. Yandek, A. I. Elegbede, A. Hinderliter, and P. F. F. Almeida, "Temperature and composition dependence of the interaction of $\delta$-lysin with ternary mixtures of sphingomyelin/cholesterol/popc," Biophysical Journal, vol. 91, no. 6, pp. 2184-2197, 2006.

[5] A. Kato, A. Tsuji, M. Yanagisawa et al., "Phase separation on a phospholipid membrane inducing a characteristic localization of DNA accompanied by its structural transition," Journal of Physical Chemistry Letters, vol. 1, no. 23, pp. 3391-3395, 2010.

[6] L. V. Schäfer and S. J. Marrink, "Partitioning of lipids at domain boundaries in model membranes," Biophysical Journal, vol. 99, no. 12, pp. L91-L93, 2010.

[7] L. V. Schäfer, D. H. de Jong, A. Holt et al., "Lipid packing drives the segregation of transmembrane helices into disordered lipid domains in model membranes," Proceedings of the National Academy of Sciences of the United States of America, vol. 108, no. 4, pp. 1343-1348, 2011.

[8] L. S. Hirst, P. Uppamoochikkal, and C. Lor, "Phase separation and critical phenomena in biomimetic ternary lipid mixtures," Liquid Crystals, vol. 38, no. 11-12, pp. 1735-1747, 2011.

[9] R. N. A. H. Lewis, W. Pohle, and R. N. McElhaney, "The interfacial structure of phospholipid bilayers: differential scanning calorimetry and Fourier transform infrared spectroscopic studies of 1,2- dipalmitoyl-sn-glycero-3-phosphorylcholine and its dialkyl and acyl-alkyl analogs," Biophysical Journal, vol. 70, no. 6, pp. 2736-2746, 1996.

[10] R. Jacobs and E. Oldfield, "Deuterium nuclear magnetic resonance investigation of dimyristoyllecithin-dipalmitoyllecithin and dimyristoyllecithin-cholesterol mixtures," Biochemistry, vol. 18, no. 15, pp. 3280-3285, 1979.

[11] W.-J. Sun, R. M. Suter, M. A. Knewtson et al., "Order and disorder in fully hydrated unoriented bilayers of gel-phase dipalmitoylphosphatidylcholine," Physical Review E, vol. 49, no. 5, pp. 4665-4676, 1994.

[12] J. Zhao, J. Wu, F. A. Heberle et al., "Phase studies of model biomembranes: complex behavior of DSPC/DOPC/Cholesterol," Biochimica et Biophysica Acta-Biomembranes, vol. 1768, no. 11, pp. 2764-2776, 2007.

[13] M. Y. El-Sayed, T. A. Guion, and M. D. Fayer, "Effect of cholesterol on viscoelastic properties of dipalmitoylphosphatidylcholine multibilayers as measured by a laser-induced ultrasonic probe," Biochemistry, vol. 25, no. 17, pp. 4825-4832, 1986.

[14] H. Pfeiffer, G. Klose, K. Heremans, and C. Glorieux, "Thermotropic phase behaviour of the pseudobinary mixtures of DPPC/C 12E5 and DMPC/C12E5 determined by differential scanning calorimetry and ultrasonic velocimetry," Chemistry and Physics of Lipids, vol. 139, no. 1, pp. 54-67, 2006.
[15] H. M. Seeger, G. Marino, A. Alessandrini, and P. Facci, "Effect of physical parameters on the main phase transition of supported lipid bilayers," Biophysical Journal, vol. 97, no. 4, pp. 1067-1076, 2009.

[16] T. Kaasgaard, C. Leidy, J. H. Crowe, O. G. Mouritsen, and K. Jørgensen, “Temperature-controlled structure and kinetics of ripple phases in one- and two-component supported lipid bilayers," Biophysical Journal, vol. 85, no. 1, pp. 350-360, 2003.

[17] J. Yang and J. Appleyard, "The main phase transition of micasupported phosphatidylcholine membranes," The Journal of Physical Chemistry B, vol. 104, no. 34, pp. 8097-8100, 2000.

[18] S. Ahmed and S. L. Wunder, "Effect of high surface curvature on the main phase transition of supported phospholipid bilayers on $\mathrm{SiO}_{2}$ nanoparticles," Langmuir, vol. 25, no. 6, pp. 3682-3691, 2009.

[19] A. W. Szmodis, C. D. Blanchette, M. L. Longo, C. A. Orme, and A. N. Parikh, "Thermally induced phase separation in supported bilayers of glycosphingolipid and phospholipid mixtures," Biointerphases, vol. 5, no. 4, pp. 120-130, 2010.

[20] G. Ohlsson, A. Tigerström, F. Höök, and B. Kasemo, "Phase transitions in adsorbed lipid vesicles measured using a quartz crystal microbalance with dissipation monitoring," Soft Matter, vol. 7, no. 22, pp. 10749-10755, 2011.

[21] P. Losada-Pérez, K. L. Jiménez-Monroy, B. van Grinsven et al., "Phase transitions in lipid vesicles detected by a complementary set of methods: heat-transfer measurements, adiabatic scanning calorimetry, and dissipation-mode quartz crystal microbalance," Physica Status Solidi A, vol. 211, no. 6, pp. 1377-1388, 2014.

[22] T. Hianik, M. Šnejdárková, L. Sokolíková et al., "Immunosensors based on supported lipid membranes, protein films and liposomes modified by antibodies," Sensors and Actuators B: Chemical, vol. 57, no. 1-3, pp. 201-212, 1999.

[23] S. Mashaghi, T. Jadidi, G. Koenderink, and A. Mashaghi, "Lipid nanotechnology," International Journal of Molecular Sciences, vol. 14, no. 2, pp. 4242-4282, 2013.

[24] J. Thoen, "High resolution adiabatic scanning calorimetry and heat capacities," in Heat Capacities: Liquids, Solutions and Vapours, E. Wilhelm and T. M. Letcher, Eds., pp. 287-306, The Royal Society of Chemistry, London, UK, 2010.

[25] D. Marsh, "Cholesterol-induced fluid membrane domains: a compendium of lipid-raft ternary phase diagrams," Biochimica et Biophysica Acta-Biomembranes, vol. 1788, no. 10, pp. 21142123, 2009.

[26] E. J. Shimshick and H. M. McConnell, "Lateral phase separation in phospholipid membranes," Biochemistry, vol. 12, no. 12, pp. 2351-2360, 1973

[27] S. Mabrey and J. M. Sturtevant, "Investigation of phase transitions of lipids and lipid mixtures by high sensitivity differential scanning calorimetry," Proceedings of the National Academy of Sciences of the United States of America, vol. 73, no. 11, pp. 38623866, 1976.

[28] R. N. McElhaney, "The use of differential scanning calorimetry and differential thermal analysis in studies of model and biological membranes," Chemistry and Physics of Lipids, vol. 30, no. 2-3, pp. 229-259, 1982.

[29] R. L. Biltonen and Q. Ye, "Kinetics of the gel-to-liquid phase transition of binary lipid bilayers using volume perturbation calorimetry," in Trends in Colloid and Interface Science VII, vol. 93 of Progress in Colloid \& Polymer Science, pp. 112-117, Springer, Darmstadt, Germany, 1993.

[30] M. Fidorra, T. Heimburg, and H. M. Seeger, "Melting of individual lipid components in binary lipid mixtures studied 
by FTIR spectroscopy, DSC and Monte Carlo simulations," Biochimica et Biophysica Acta-Biomembranes, vol. 1788, no. 3, pp. 600-607, 2009.

[31] I. P. Sugár, T. E. Thompson, and R. L. Biltonen, "Monte Carlo simulation of two-component bilayers: DMPC/DSPC mixtures," Biophysical Journal, vol. 76, no. 4, pp. 2099-2110, 1999.

[32] E. I. Michonova-Alexova and I. P. Sugár, "Component and state separation in DMPC/DSPC lipid bilayers: A Monte Carlo Simulation Study," Biophysical Journal, vol. 83, no. 4, pp. 18201833, 2002.

[33] J. Ehrig, E. P. Petrov, and P. Schwille, "Phase separation and near-critical fluctuations in two-component lipid membranes: Monte Carlo simulations on experimentally relevant scales," New Journal of Physics, vol. 13, Article ID 045019, 2011.

[34] J. Leys, P. Losada-Pérez, C. Glorieux, and J. Thoen, "Application of a novel type of adiabatic scanning calorimeter for highresolution thermal data near the melting point of gallium," Journal of Thermal Analysis and Calorimetry, vol. 117, no. 1, pp. 173-187, 2014.

[35] J. Leys, P. Losada-Pérez, E. Slenders, C. Glorieux, and J. Thoen, "Investigation of the melting behavior of the reference materials biphenyl and phenyl salicylate by a new type adiabatic scanning calorimeter," Thermochimica Acta, vol. 582, pp. 68-76, 2014.

[36] C. A. Keller and B. Kasemo, "Surface specific kinetics of lipid vesicle adsorption measured with a quartz crystal microbalance," Biophysical Journal, vol. 75, no. 3, pp. 1397-1402, 1998.

[37] W. Wagner and A. Pruß, "The IAPWS formulation 1995 for the thermodynamic properties of ordinary water substance for general and scientific use," Journal of Physical and Chemical Reference Data, vol. 31, no. 2, pp. 387-535, 2002.

[38] D. P. Kharakoz, M. S. Panchelyuga, E. I. Tiktopulo, and E. A. Shlyapnikova, "Critical temperatures and a critical chain length in saturated diacylphosphatidylcholines: calorimetric, ultrasonic and Monte Carlo simulation study of chain-melting/ordering in aqueous lipid dispersions," Chemistry and Physics of Lipids, vol. 150, no. 2, pp. 217-228, 2007.

[39] K. Denolf, G. Cordoyiannis, C. Glorieux, and J. Thoen, "Effect of nonmesogenic impurities on the liquid crystalline phase transitions of octylcyanobiphenyl," Physical Review E, vol. 76, no. 5, Article ID 051702, 2007.

[40] C. S. P. Tripathi, P. Losada-Pérez, J. Leys, G. Cordoyiannis, C. Glorieux, and J. Thoen, "Evidence from adiabatic scanning calorimetry for the Halperin-Lubensky-Ma effect at the NSmA phase transitions in mixtures of $7 \mathrm{OCB}$ +heptane with an injected SmA phase," The European Physical Journal E, vol. 35, no. 7, article 54, 2012.

[41] Y. Jing, H. Trefna, M. Persson, B. Kasemo, and S. Svedhem, "Formation of supported lipid bilayers on silica: relation to lipid phase transition temperature and liposome size," Soft Matter, vol. 10, no. 1, pp. 187-195, 2014.

[42] M. A. Meyers and K. K. Chawla, Mechanical Behavior of Materials, Cambridge University Press, Cambridge, UK, 2nd edition, 2009.

[43] A. P. Serro, A. Carapeto, G. Paiva, J. P. S. Farinha, R. Colaço, and B. Saramago, "Formation of an intact liposome layer adsorbed on oxidized gold confirmed by three complementary techniques: QCM-D, AFM and confocal fluorescence microscopy," Surface and Interface Analysis, vol. 44, no. 4, pp. 426-433, 2012.

[44] R. Koynova and M. Caffrey, "Phases and phase transitions of the phosphatidylcholines," Biochimica et Biophysica Acta-Reviews on Biomembranes, vol. 1376, no. 1, pp. 91-145, 1998.
[45] B. Tenchov, R. Koynova, and G. Rapp, "New ordered metastable phases between the gel and subgel phases in hydrated phospholipids," Biophysical Journal, vol. 80, no. 4, pp. 1873-1890, 2001.

[46] C. Leidy, T. Kaasgaard, J. H. Crowe, O. G. Mouritsen, and K. Jørgensen, "Ripples and the formation of anisotropic lipid domains: imaging two-component supported double bilayers by atomic force microscopy," Biophysical Journal, vol. 83, no. 5, pp. 2625-2633, 2002.

[47] U. Bernchou, H. Midtiby, J. H. Ipsen, and A. C. Simonsen, "Correlation between the ripple phase and stripe domains in membranes," Biochimica et Biophysica Acta-Biomembranes, vol. 1808, no. 12, pp. 2849-2858, 2011.

[48] E. J. Luna and H. M. McConnell, "Multiple phase equilibria in binary mixtures of phospholipids," Biochimica et Biophysica Acta-Biomembranes, vol. 509, no. 3, pp. 462-473, 1978.

[49] R. L. Biltonen and D. Lichtenberg, "The use of differential scanning calorimetry as a tool to characterize liposome preparations," Chemistry and Physics of Lipids, vol. 64, no. 1-3, pp. 129-142, 1993. 

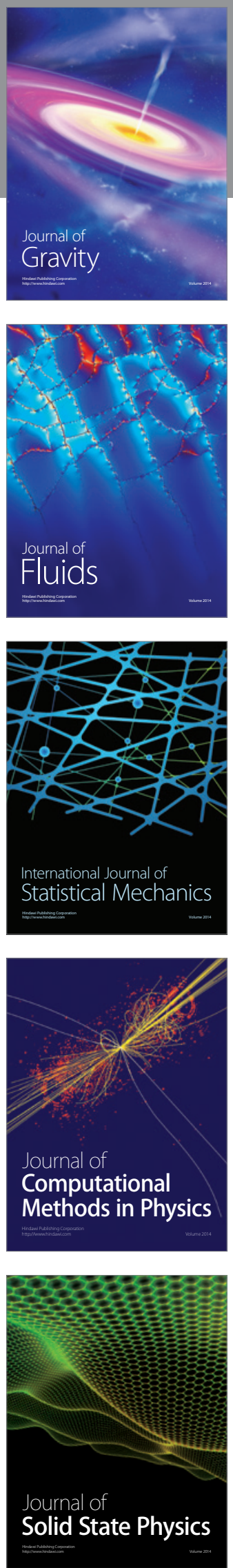

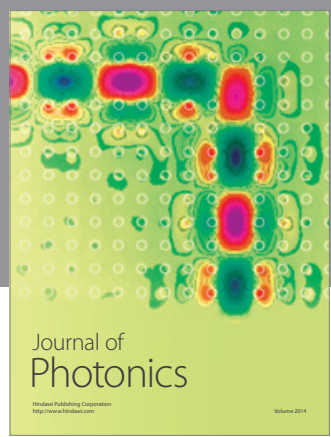

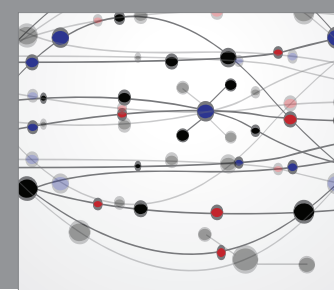

The Scientific World Journal

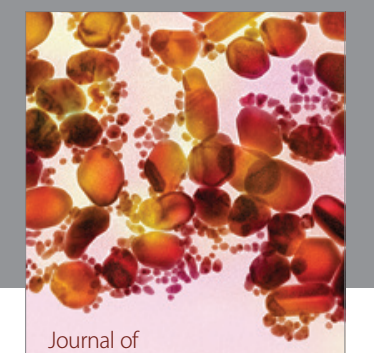

Soft Matter
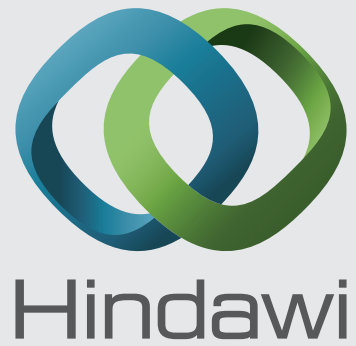

Submit your manuscripts at

http://www.hindawi.com
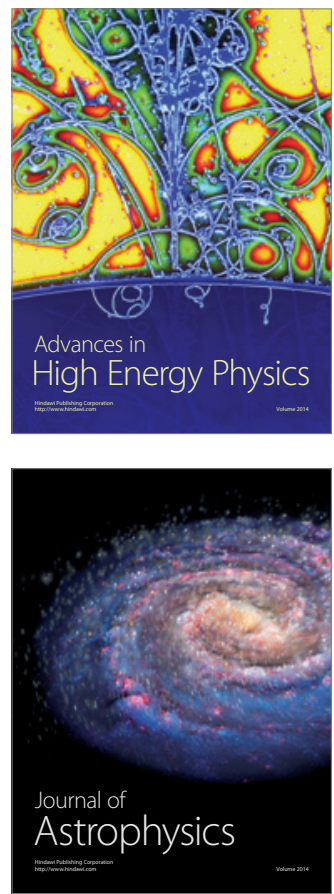
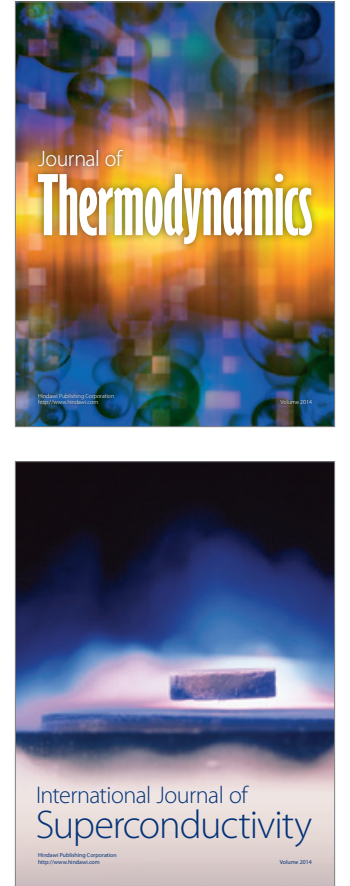
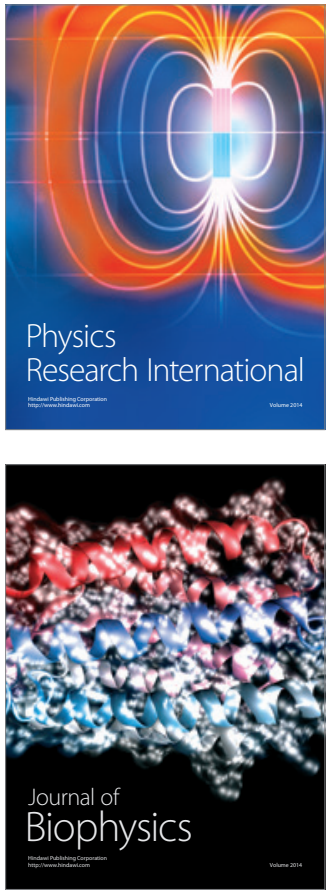
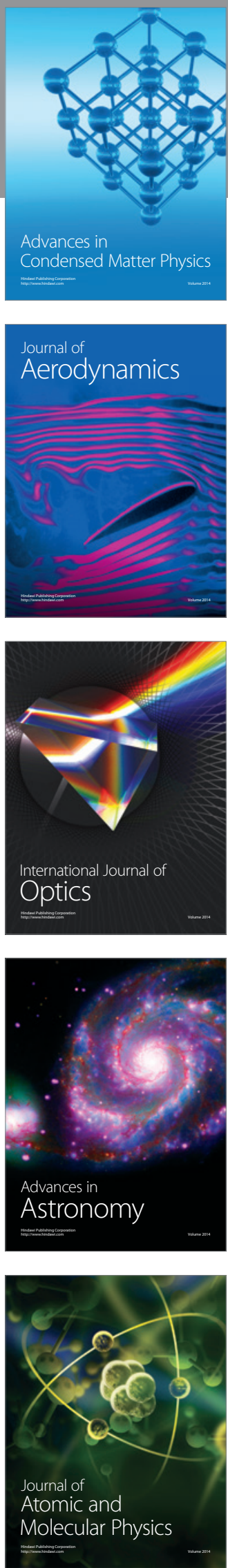